\title{
Measurement of optical seeing on the high antarctic plateau
}

\author{
R.D. Marks ${ }^{1}$, J. Vernin ${ }^{2}$, M. Azouit ${ }^{2}$, J.F. Manigault ${ }^{2}$, and C. Clevelin ${ }^{3}$ \\ 1 Joint Australian Centre for Astrophysical Research in Antarctica, School of Physics, University of New South Wales, Sydney \\ 2052, Australia \\ 2 Département d'Astrophysique de l'Université de Nice, URA 709 du CNRS, F-06108 Nice Cedex 2, France \\ 3 Antarctic Support Associates, PO Box 300, Englewood CO 80112, U.S.A.
}

Received November 21; accepted July 21, 1998

\begin{abstract}
Results from the 1995 season of site-testing experiments at the South Pole are presented, in which the seeing was measured using balloon-borne microthermal probes. Our analysis shows a marked division of the atmosphere into two characteristic regions: (i) a highly turbulent boundary layer $(0-220 \mathrm{~m})$ associated with a strong temperature inversion and wind shear, and (ii) a very stable free atmosphere. The mean seeing, averaged over 15 balloon flights, was measured to be $1.86^{\prime \prime}$, of which the free atmosphere component was only $0.37^{\prime \prime}$. The seeing from $\sim 200 \mathrm{~m}$ upward is superior to the leading mid-latitude sites (e.g. Fuchs 1995; Roddier et al. 1990) by almost a factor of two. The results are in good agreement with optical seeing data obtained by a differential image motion monitor on three of the five occasions when the two measurements were performed simultaneously. The boundary layer winds are of katabatic origin, and so we may consider the possibility of exceptional seeing conditions from surface level at other locations on the plateau such as Domes $\mathrm{A}$ and $\mathrm{C}$, where there is little or no katabatic wind. In addition, the proximity of the optical turbulence to the focus of a telescope situated at ground level is a highly favourable situation for the use of adaptive optics, since the wavefront spatial coherence scale is related to the altitude of the turbulent layers producing the image distortion. Some comparisons are made between the relevant adaptive optics parameters measured at the South Pole and Cerro Paranal, one of the best mid-latitude sites.
\end{abstract}

Key words: atmospheric effects - balloons instrumentation: miscellaneous - methods: observational — site testing

Send offprint requests to: $\mathrm{R}$. Marks

\section{Introduction}

The likely characteristics of the high antarctic plateau for astronomy have been widely discussed, and are described in greater detail elsewhere (e.g. Burton 1996). This experiment forms part of a continuing program of site-testing in Antarctica, being conducted by groups from the University of New South Wales (Australia), the Université de Nice (France) and the Center for Astrophysical Research in Antarctica (U.S.A.), of which the aim is to determine as completely as possible the observing conditions at the South Pole, before performing measurements at more remote sites on the plateau. Several other experiments are in progress, the results from some of which have been reported (Ashley et al. 1996; Nguyen et al. 1996).

The results presented here form the second part of a campaign to determine the optical seeing at the South Pole, and its variation with altitude, by direct measurement of the thermal fluctuations associated with the atmospheric turbulence. These can be directly related to refractive index variations, which are the source of atmospheric seeing. In the first part of this experiment (Marks et al. 1996) we observed very strong optical turbulence close to the surface $\left(0.64^{\prime \prime}\right.$ on average in the lowest $\left.27 \mathrm{~m}\right)$, compared with similar measurements performed at midlatitude sites (Vernin \& Tuñon-Muñóz 1994; ESO-VLT working group 1987), which put the seeing contribution from this region at $\lesssim 0.1^{\prime \prime}$. Individual measurements were highly variable, and often indicated a significant decrease in optical turbulence with height above the surface. It became clear from these results that determining the vertical extent of turbulence in the boundary layer was of prime importance in characterising the seeing at the site.

In our second season, balloon-borne microthermal sensors were used to obtain integrated values of the seeing over the entire atmosphere, as well as to observe the altitude profile of the optical turbulence. The results of fifteen balloon flights are discussed here, including the relation of 
the turbulence profile to simultaneously measured temperature and wind velocity gradients. We use the observed correlation between these quantities to speculate on the likely conditions at other sites higher on the plateau.

The strong concentration of optical turbulence in the boundary layer is a highly favourable situation for the use of image correction techniques, and in the final section we quantify some of the important site parameters in adaptive optics, for comparison with the values obtained under the conditions found at mid-latitude sites.

\section{Principles of microthermal measurement of seeing}

The pairs of microthermal sensors used in this experiment measure the temperature structure function associated with the turbulence:

$D_{\mathrm{T}}(\boldsymbol{\rho}, h)=\left\langle(T(\mathbf{r}, h)-T(\mathbf{r}+\boldsymbol{\rho}, h))^{2}\right\rangle$

where $\boldsymbol{\rho}$ is the separation of the sensors and $h$ is the altitude. Assuming fully developed turbulence according to the theory of Kolmogorov (Tatarski 1961), with $|\boldsymbol{\rho}|$ between the inner and outer scales of the turbulent motion (the "inertial sub- range"), we can obtain the corresponding refractive index structure parameter using (Roddier 1981):

$C_{\mathrm{N}}^{2}(h)=\left(80.10^{-6} \frac{P(h)}{T(h)^{2}}\right)^{2} \rho^{-2 / 3} D_{\mathrm{T}}(\boldsymbol{\rho}, h)$

where $P(h)$ is the pressure and $T(h)$ the temperature.

The seeing quality is commonly described in terms of the "Fried parameter" (Fried 1966):

$r_{0}=\left(0.423 k^{2} \sec \gamma \int_{h_{0}}^{\infty} C_{\mathrm{N}}^{2}(h) \mathrm{d} h\right)^{-3 / 5}$

where $k$ is the wavenumber, $\gamma$ the zenith angle and $h_{0}$ the height of the telescope.

The Fried parameter is interpreted as the spatial coherence scale of the atmosphere. Atmospheric turbulence reduces the image resolution from $\mathrm{O}(\lambda / D)$, where $D$ is the telescope diameter, to $\mathrm{O}\left(\lambda / r_{0}\right)$. The exact expression for the full width at half maximum of the "seeing disk" is (Roddier 1981; Dierickx 1992):

$\varepsilon_{\mathrm{fwhm}}=0.98 \frac{\lambda}{r_{0}}$.

\section{Results}

Fifteen successful balloon launches were performed between June 20 and August 18, 1995, during the polar night. Each balloon payload contained our microthermal sensors, as well as a Vaisala radiosonde which supplied the required pressure and temperature measurements. In addition, the launches were timed to coincide with weather balloon flights, from which we were able to obtain wind velocity profiles.

The sampling rate of the radiosondes resulted in a vertical resolution of approximately $5-6 \mathrm{~m}$. The mean seeing results were obtained by constructing a set of average $C_{\mathrm{N}}^{2}$ values at standard altitudes, in $5 \mathrm{~m}$ steps, interpolating between the nearest raw data points on either side of each standard level. The average seeing produced by any layer of the atmosphere may then be calculated simply by varying the limits of the integral in Eq. (3). Inspection of the raw data indicated that these altitude increments were small enough for linear interpolation to be an accurate approximation to the $C_{\mathrm{N}}^{2}$ profile. Unless otherwise stated, all calculations are performed using a wavelength of $0.5 \mu \mathrm{m}$ and zenith angle $\gamma=0^{\circ}$.

Due to the difficulty of the launch procedure in polar winter conditions, the tether length between balloon and sonde at launch was only $10-20 \mathrm{~m}$ for most flights, with a reel attached to gradually pay out a further $30 \mathrm{~m}$ during the early part of the ascent. It is possible that the turbulent wake of the balloon had a minor effect on the data before the tether rolled out to its full extent $(\sim 50 \mathrm{~m})$, especially in regions of low wind speed (i.e. when the balloon ascends almost vertically above the sonde), and hence the stated values of the seeing should be considered as upper limits.

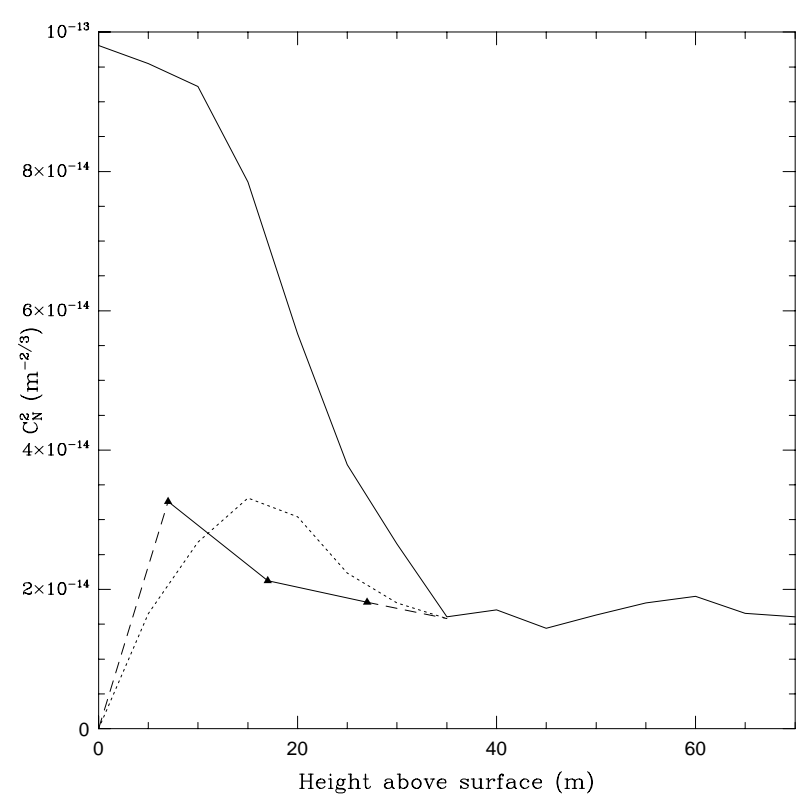

Fig. 1. Average $C_{\mathrm{N}}^{2}$ profile up to a height of $70 \mathrm{~m}$, calculated from the 15 balloon sondes. Included are the two methods used to determine the lower altitude limit of the data, and extrapolate down to the surface. The mean $C_{\mathrm{N}}^{2}$ measurements from the $27 \mathrm{~m}$-high tower (Marks et al. 1981) are indicated by the triangles, with extrapolations from $0-7 \mathrm{~m}$ and from $27-35 \mathrm{~m}$ shown by the dashed lines. The dotted line shows the average values obtained by editing the individual $C_{\mathrm{N}}^{2}$ profiles in the $0-40 \mathrm{~m}$ range, with reference to the corresponding wind and temperature profiles 
Table 1. Summary of integrated seeing and boundary layer data, from 16 balloon launches between 20 June and 18 August 1995. The "free atmosphere" refers to the entire atmosphere excluding the boundary layer. Values are quoted for a wavelength of $0.5 \mu \mathrm{m}$

\begin{tabular}{|c|c|c|c|c|c|c|}
\hline Measurement & Mean & Std. Dev. & Median & Best $25 \%$ & Best & Worst \\
\hline \multicolumn{7}{|l|}{ Seeing (arcseconds) } \\
\hline -total & $1.86 \pm 0.02$ & 0.75 & 1.6 & 1.0 & $0.8 \pm 0.1$ & $3.1 \pm 0.2$ \\
\hline -free atmosphere & 0.37 & 0.07 & 0.32 & 0.29 & 0.23 & 0.52 \\
\hline \multicolumn{7}{|l|}{$r_{0}(\mathrm{~cm})$} \\
\hline- total & $5.48 \pm 0.05$ & 3.40 & 6.4 & 9.9 & 12.3 & 3.3 \\
\hline -free atmosphere & 27.20 & 7.16 & 28.18 & 34.77 & 44.58 & 23.33 \\
\hline Boundary layer height $(\mathrm{m})$ & 220 & 70 & 190 & 165 & 120 & 275 \\
\hline
\end{tabular}

In addition, the first $5-10 \mathrm{~s}$ of each flight's data were contaminated by large temperature fluctuations associated with the launch procedure, and it was expected that the seeing contribution from the lowest $30-50 \mathrm{~m}$ would be greatly overestimated due to this effect. Two methods were used to try to estimate as accurately as possible the $C_{\mathrm{N}}^{2}$ profile in this region. The first was to examine the raw data for individual flights in detail, with reference to the temperature and wind velocity gradients (see Sect. 3.4), and estimate the correct $C_{\mathrm{N}}^{2}$ values for each flight, in the lowest $50 \mathrm{~m}$. The second method involved using the 1994 study of lower-boundary layer turbulence (Marks et al. 1996), from which we have long-term average $C_{\mathrm{N}}^{2}$ values up to a height of $27 \mathrm{~m}$. Comparing this with the corresponding average radiosonde data clearly indicated the lower limit of validity of the balloon data, which could then be interpolated down to ground level using the 1994 values. The results from both of these analyses are shown in Fig. 1, together with the unmodified average $C_{\mathrm{N}}^{2}$ profile. It is clear that the radiosonde data is valid upward from around $40 \mathrm{~m}$. Below this level, the two methods described provide very consistent estimates of the average $C_{\mathrm{N}}^{2}$ values.

A further difficulty was that most of the balloons reached an altitude of only $12-15 \mathrm{~km}$, rather than the usual $25+\mathrm{km}$, due to the reduced strength of the balloon material at very low temperatures. Thus any turbulence above $15 \mathrm{~km}$ was not measured. However, due to the very low air pressure, the optical turbulence at this altitude is generally extremely low, and the $C_{\mathrm{N}}^{2}$ measurements invariably dropped to around the $10^{-18}-10^{-19} \mathrm{~m}^{-2 / 3}$ level by the end of each flight. This trend continued in the one flight that reached an altitude of $>20 \mathrm{~km}$. Integrating up to $25 \mathrm{~km}$ using an artificial $C_{\mathrm{N}}^{2}$ profile based on weather balloon pressure and temperature data from the NOAA weather balloons (see Eq. 2) indicated that the unsampled region of the atmosphere contributed less than $0.01^{\prime \prime}$ to the seeing.

\subsection{Summary of results}

The seeing measurements from the 15 flights are summarised in Table 1. The weather observer's notes from the time of each launch indicate that 10 of the 15 flights took place in clear conditions, with some scattered cloud present on the other 5 days. The mean total integrated seeing was measured to be $1.86 \pm 0.02^{\prime \prime}$. The quoted uncertainty represents the slight discrepancy between the results from the two methods (described above) used to estimate the contribution from $0-40 \mathrm{~m}$. The average free atmosphere contribution of $0.37^{\prime \prime}$ (corresponding to $\left.r_{0}=27.2 \mathrm{~cm}\right)$ represents only $\sim 7 \%$ of the integrated optical turbulence $\left(\int C_{\mathrm{N}}^{2}(h) \mathrm{d} h\right)$.

The range of total seeing values is very large $\left(\sigma=0.75^{\prime \prime}\right)$, and sub-arcsecond seeing was measured from ground level in 3 of the 15 flights. The free atmosphere contribution, on the other hand, was relatively constant throughout the season $\left(\sigma=0.07^{\prime \prime}\right)$, which indicates that the variability is largely due to fluctuations in boundary layer turbulence. Figure 2 shows the statistical distribution of total and free atmosphere integrated seeing results. The intense and highly variable boundary layer signal agrees with the results of the 1994 experiment (Marks et al. 1996), where the seeing contribution from just the lowest $27 \mathrm{~m}$ was measured to be $0.64^{\prime \prime}, \sigma=0.40^{\prime \prime}$.

The height of the boundary layer is $220 \mathrm{~m}$ on average. The upper limit of the boundary layer was defined as the lowest height, $h_{0}$, at which successive calculations of the integrated seeing (Eqs. (3)-(4)) varied according to:

$\varepsilon_{h_{0}+1}^{\infty}-\varepsilon_{h_{0}}^{\infty} \leq 0.001^{\prime \prime}$

where the sub- and super-scripts refer to the limits of the integration in Eq. (3). In all cases, this corresponded closely to the limit of the steeply increasing section of the temperature profile, although the temperature inversion occasionally continued more weakly for a further $100 \mathrm{~m}$ or so.

It is worth noting the wavelength dependence of $\varepsilon$ : from Eqs. (3-4), $\varepsilon \propto \lambda^{-1 / 5}$, and so, for example, at a 
wavelength of $2.4 \mu \mathrm{m}$, the corresponding values are $1.36^{\prime \prime}$ for the full atmosphere and $0.27^{\prime \prime}$ for the free atmosphere. This particular wavelength is of significance since it is in the so-called "cosmological window": a waveband corresponding to a natural minimum in airglow emission, and at which the sky brightness due to thermal emission at the South Pole is a factor of 10-100 lower than at any other ground-based site (Ashley et al. 1996).

\subsection{Comparison with H-DIMM observations}

A modified version of a differential image motion monitor, known as an "H- DIMM" (Bally et al. 1996) was also in place at the South Pole during 1995. This instrument consisted of a $60 \mathrm{~cm}$ telescope with a multiple-aperture mask, and is essentially similar in principle to the standard DIMM as described by Sarazin \& Roddier (1996).

Seeing measurements were taken by the H-DIMM near-simultaneously with a microthermal balloon flight on 5 occasions during the season. The results are compared in Fig. 3. The correlation between the two experiments is reasonably good for three of the flights, while for the other two, the microthermal data gives a significantly lower value for the FWHM seeing than does the H-DIMM. These discrepancies are most probably due to serious disturbances in the H-DIMM images caused by internal heaters being inadvertently left on during many of the observations, a problem which was not noticed until after the completion of the experiment. These distortions were thought to be largely confined to one particular region of the image data, which was removed during the reduction process. However, it is possible that some effect of this heating was present across the rest of the image, which may have artificially elevated the derived seeing. A small residual effect of this nature would be most noticeable at times of relatively low atmospheric seeing, as was the case for the two observations in question.

\subsection{Individual flight characteristics; relation of boundary layer seeing to atmospheric parameters}

The vertical profile of the optical turbulence at the South Pole is markedly different from that measured using similar techniques at other sites around the world. Figure 4 is a plot of $C_{\mathrm{N}}^{2}$ vs. altitude for three of the balloon launches, and illustrates the conditions typically observed. Potential temperature $^{1}$ and wind velocity gradients are also included in the boundary layer profiles (Figs. 4a,c,e). Note that different scales are used on the $C_{\mathrm{N}}^{2}$ axes for the three boundary layer plots.

\footnotetext{
${ }^{1}$ Potential temperature is defined as: $\theta(h)=$ $T(h)\left(\frac{P(h)}{1000}\right)^{-0.288}$.
}
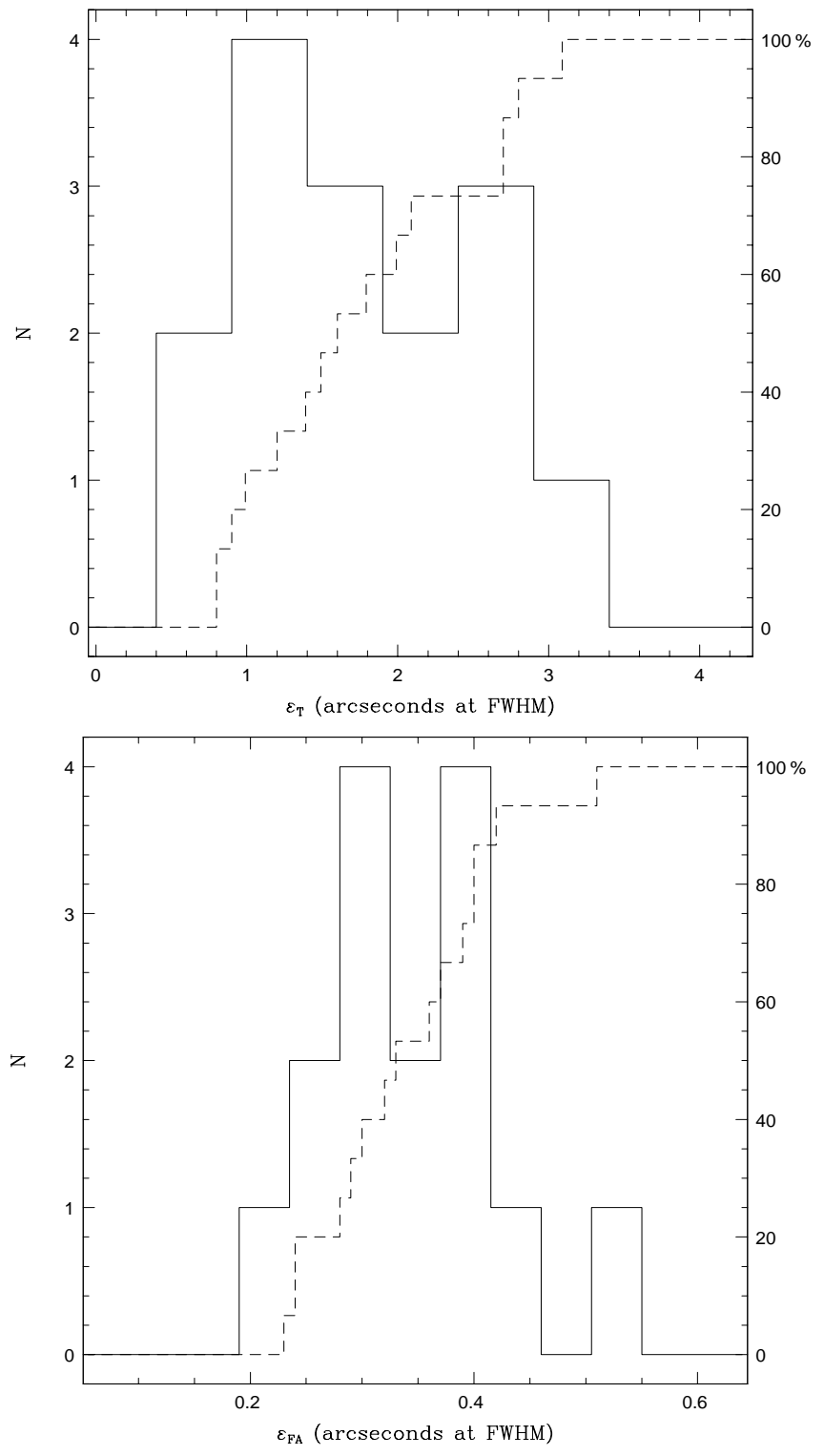

Fig. 2a and b. Seeing data statistics for the 15 balloon sondes launched in 1995: a) total (from ground level), b) free atmosphere

The boundary layer turbulence structure is quite complex, and contains several strong $C_{\mathrm{N}}^{2}$ peaks, generally occurring in layers around $10-20 \mathrm{~m}$ in thickness. The most intense of these are often concentrated in two regions; one close to the surface (up to $50-100 \mathrm{~m}$ ), and another closer to the top of the inversion layer $(\sim 200 \mathrm{~m})$. This feature is clearly evident in Figs. 4a,c, and was observed in many of the other flights. It corresponds to a "two-tiered" temperature inversion, as indicated by the secondary rise in $\mathrm{d} \theta / \mathrm{d} z$ in each of these flights.

The layers of intense optical turbulence, and its overall rapid decrease with altitude, are strongly correlated with simultaneous sharp fluctuations in $\mathrm{d} \theta / \mathrm{d} z$ and $|\mathrm{d} \boldsymbol{U} / \mathrm{d} z|$, as can be seen clearly in the three flights shown. Figure 5 

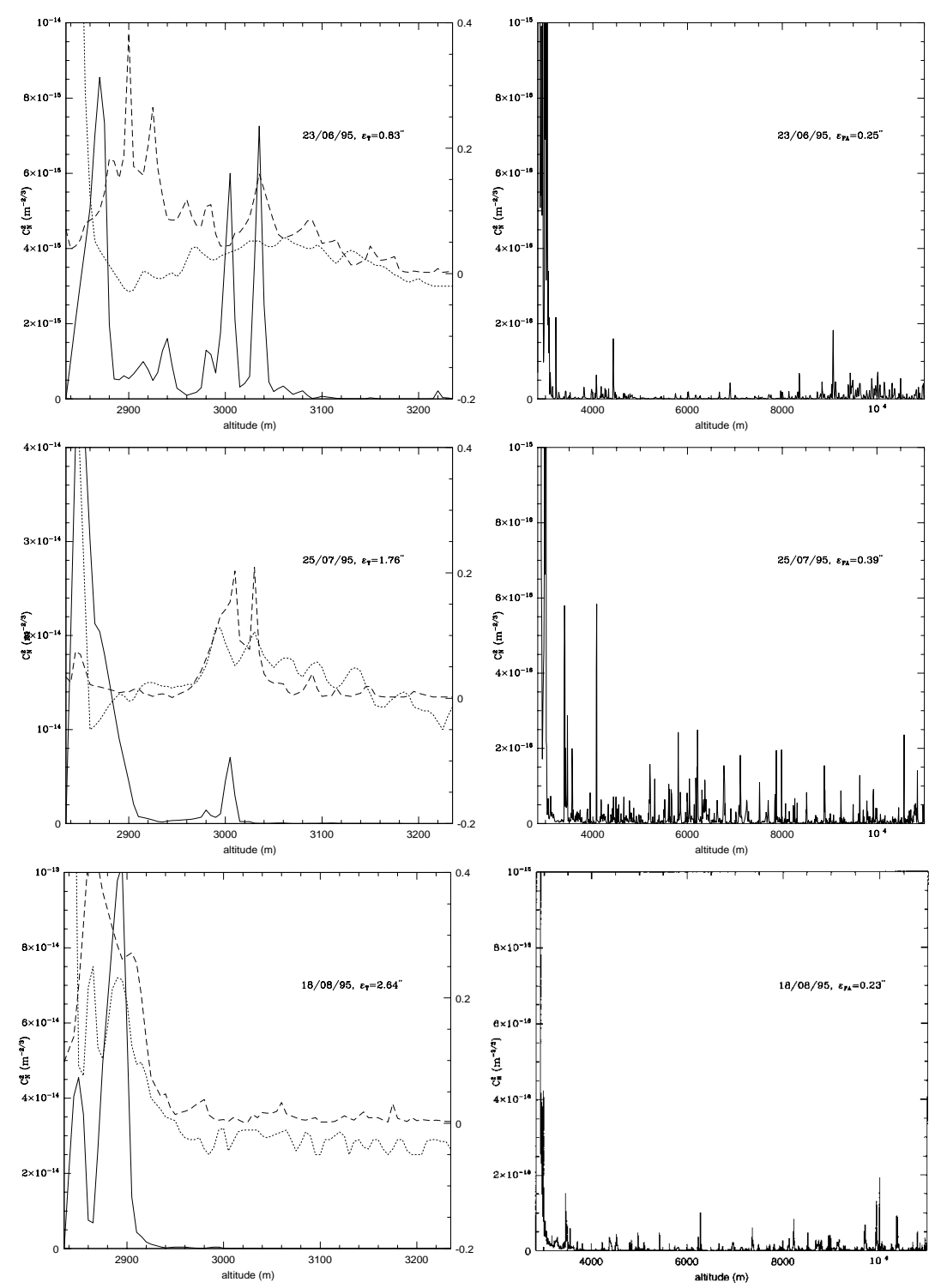

Fig. 4a-f. $C_{\mathrm{N}}^{2}$ vs. altitude: a-b) for 23/06/95, c-d) for 21/07/95, e-f) for 18/08/95. The boundary layer plots include potential temperature gradient, $|\mathrm{d} \theta / \mathrm{d} z|$ (dashed lines) and wind velocity gradient, $|\mathrm{d} \boldsymbol{U} / \mathrm{d} z|$ (dotted lines). The axes on the right of each plot show $|\mathrm{d} \theta / \mathrm{d} z|$, in units of ${ }^{\circ} \mathrm{C} / \mathrm{m}^{-1}$. Units for $|\mathrm{d} \boldsymbol{U} / \mathrm{d} z|$ are not shown; it is approximately equal to 0.5 (RHS axis scale) $\mathrm{m} \mathrm{s}^{-1}$, offset by -0.05 for clarity

shows the average profiles of these quantities along with the $C_{\mathrm{N}}^{2}$ measurements. The mean temperature inversion magnitude is $\sim 25^{\circ} \mathrm{C}$. These observations agree with the known requirements for optical turbulence: the presence of both a significant temperature gradient and mechanical turbulence produced by wind shear. In other words, it is the combination of a strong temperature inversion and a wind velocity gradient which produces such a strong seeing contribution close to the surface, as can be seen clearly in Fig. 5.

The occurrence of sub-arcsecond seeing from ground level (observed on three occasions) tended to coincide with a shift in wind direction away from the bearing of the usual katabatic flow (between grid $\mathrm{N}$ and $\mathrm{E}$ ) toward the SE quadrant, with a corresponding increase in surface level temperature and decrease in wind speed. The temperature during the best seeing conditions observed $\left(0.8^{\prime \prime}\right)$ was $-42^{\circ} \mathrm{C}$, about $18^{\circ} \mathrm{C}$ above average. These influxes of relatively warm air from the coast onto the plateau occur very occasionally throughout the winter. The markedly improved seeing that occurs during these events highlights the importance of the katabatic wind to the generation of optical turbulence in the boundary layer.

Relatively poor seeing $\left(>2.5^{\prime \prime}\right)$, tended to occur during fairly typical wind and temperature conditions. However, the three worst boundary layer seeing measurements coincided with a very pronounced "double inversion", as 


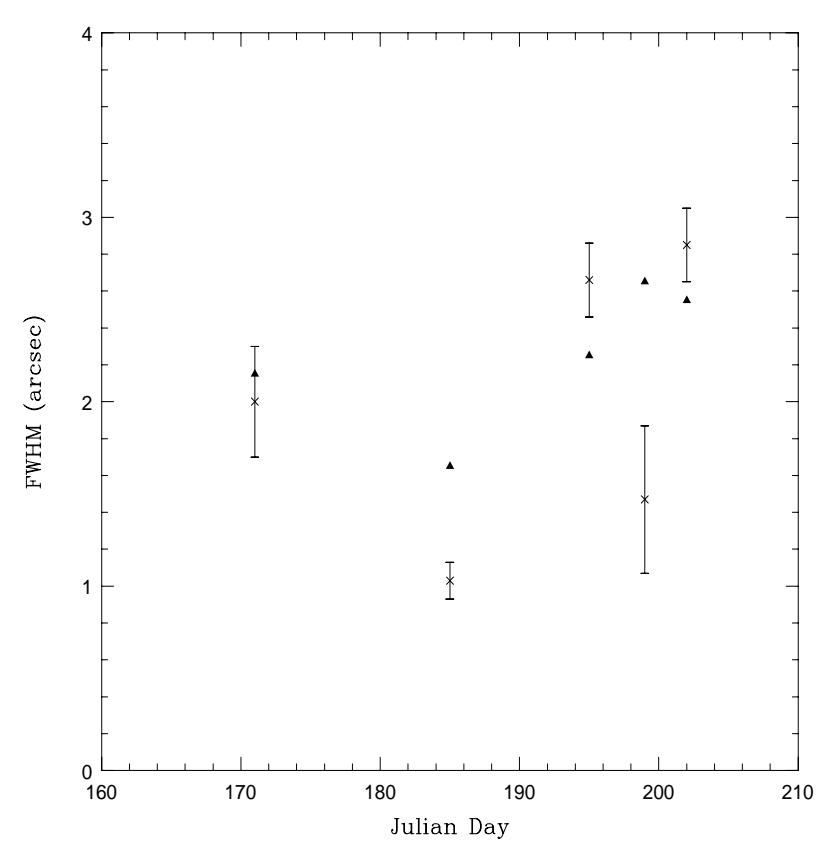

Fig. 3. Comparison of H-DIMM (triangles) and microthermal (crosses) seeing measurements on the five occasions that the two experiments were performed simultaneously, from 23 June to 21 July, 1995. The error bars derive from the uncertainty in the $C_{\mathrm{N}}^{2}$ values from $0-40 \mathrm{~m}$

described previously, with very intense $C_{\mathrm{N}}^{2}$ peaks in the upper part of the boundary layer.

In all but one of the flights, the free atmosphere turbulence was very weak, with the strongest peaks usually around two orders of magnitude weaker than those measured in the boundary layer. There was very little tropopause instability observed in any of the data, with occasional layers of increased optical turbulence not restricted to any particular region of the atmosphere.

The conditions for wind-generated turbulence are often defined in terms of the Richardson number:

$$
\begin{aligned}
R_{\mathrm{i}} & =\frac{g}{\theta} \frac{(\mathrm{d} \theta / \mathrm{d} z)}{(\mathrm{d} \boldsymbol{U} / \mathrm{d} z)^{2}} \\
& <\frac{1}{4} .
\end{aligned}
$$

Equation (5) is usually stated as the criterion for the development of turbulence. This inequality was calculated for all flights, and observed to be consistent with the $C_{\mathrm{N}}^{2}$ measurements in the lower part of the boundary layer as a whole. However, it did not appear to describe very well the intense individual turbulent layers, which often coincided with large positive values of $\mathrm{d} \theta / \mathrm{d} z$. In the free atmosphere, the occasional layers of relatively large $C_{\mathrm{N}}^{2}$ were often associated with dips in $R_{\mathrm{i}}$ below the critical value of $1 / 4$, as shown in the example of Fig. 6. Any features not coinciding with a decrease in $R_{\mathrm{i}}$ generally occurred in thin layers with a sharp rise in $\theta$ of up to $1-2^{\circ} \mathrm{C}$.

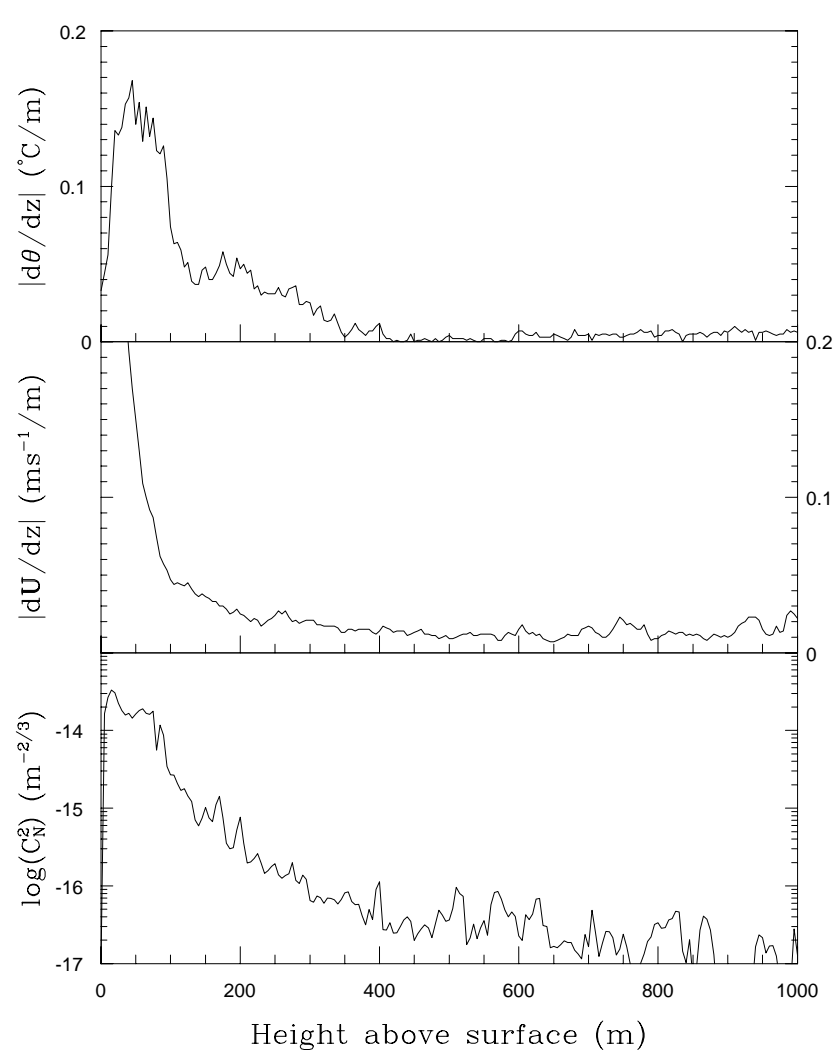

Fig. 5. Average $C_{\mathrm{N}}^{2},|\mathrm{~d} \theta / \mathrm{d} z|$, and $|\mathrm{d} \boldsymbol{U} / \mathrm{d} z|$ profiles up to a height of $1 \mathrm{~km}$ above the surface. Wind velocity data were obtained from weather balloon launches performed almost simultaneously with our flights

Most of the free atmosphere seeing was caused by these turbulent layers. The flights where no sharp peaks in $C_{\mathrm{N}}^{2}$ were observed ( 6 out of 15 , including Figs. $4 \mathrm{~b}, \mathrm{f}$ ) gave $\varepsilon_{\mathrm{FA}}=0.23-0.26^{\prime \prime}$. Further analysis of the long-term meteorological records, with regard to the behaviour of $R_{\mathrm{i}}$ and $\mathrm{d} \theta / \mathrm{d} z$, will therefore be useful in determining the frequency of the best free atmosphere seeing conditions at the South Pole.

The $C_{\mathrm{N}}^{2}$ measurements are in broad agreement with acoustic soundings performed at the South Pole since 1975 (e.g. Neff 1981), as well as other analyses of the meteorological data (Schwerdtfeger 1984; Gillingham 1993). While the acoustic backscatter measurements of $C_{\mathrm{N}}^{2}$ had a lower vertical resolution than the microthermal balloon sondes, they did illustrate the same features, i.e. a complex turbulence structure, often split into two layers, extending up to an altitude of $200-300 \mathrm{~m}$, with a sharply defined upper limit, and much weaker activity thereafter.

\subsection{Comparison with other sites}

In contrast to the conditions observed at the South Pole, while the $C_{\mathrm{N}}^{2}$ profiles measured at mid-latitude sites (e.g. Roddier et al. 1990; Vernin \& Muñoz-Tunón 1994) often 
Table 2. Comparison of seeing conditions at the South Pole and some of the world's major observatory sites. FA, BL and SL refer to the free atmosphere, boundary layer and surface layer, respectively

\begin{tabular}{|c|c|c|c|c|c|c|c|}
\hline Site & $\begin{array}{l}\text { Altitude } \\
{[\mathrm{m}]}\end{array}$ & $\begin{array}{l}\text { Total seeing } \\
{\left[{ }^{\prime \prime}\right]}\end{array}$ & $\begin{array}{l}\text { FA seeing } \\
{\left[{ }^{\prime \prime}\right]}\end{array}$ & $\begin{array}{l}\text { BL seeing } \\
{\left[{ }^{\prime \prime}\right]}\end{array}$ & $\begin{array}{l}\text { SL seeing/ range } \\
{\left[{ }^{\prime \prime}, \mathrm{m}\right]}\end{array}$ & $\begin{array}{l}\text { BL height } \\
{[\mathrm{m}]}\end{array}$ & Reference \\
\hline South Pole & 2835 & 1.86 & 0.37 & 1.78 & $0.64,27$ & 220 & this work, Marks et al. 1996 \\
\hline $\begin{array}{l}\text { Cerro Paranal, } \\
\text { Chile }\end{array}$ & 2500 & $\begin{array}{l}0.64(\text { median }) \\
0.73\end{array}$ & $\begin{array}{l}- \\
0.4\end{array}$ & $-\overline{0}-5$ & - & $\begin{array}{l}- \\
2000\end{array}$ & $\begin{array}{l}\text { Murtagh \& Sarazin } 1993 \\
\text { Fuchs } 1995\end{array}$ \\
\hline $\begin{array}{l}\text { La Silla, } \\
\text { Chile }\end{array}$ & 2400 & $\begin{array}{l}0.97 \\
0.87\end{array}$ & $\begin{array}{l}0.31 \\
-\end{array}$ & $\begin{array}{l}0.85 \\
-\end{array}$ & $\begin{array}{l}0.15,30 \\
-\end{array}$ & $\begin{array}{l}800-1000 \\
-\end{array}$ & $\begin{array}{l}\text { ESO-VLT report } 1987 \\
\text { Murtagh \& Sarazin } 1993\end{array}$ \\
\hline $\begin{array}{l}\text { Mauna Kea, } \\
\text { Hawaii }\end{array}$ & 4200 & 0.74 & 0.46 & 0.52 & - & - & Roddier et al. 1990 \\
\hline $\begin{array}{l}\text { La Palma, } \\
\text { Canary Is. }\end{array}$ & 2100 & 0.96 & 0.40 & 0.73 & $0.07,12$ & $1-2000$ & Vernin \& Muñoz-Tũ̃ón 1992, 1994 \\
\hline
\end{tabular}

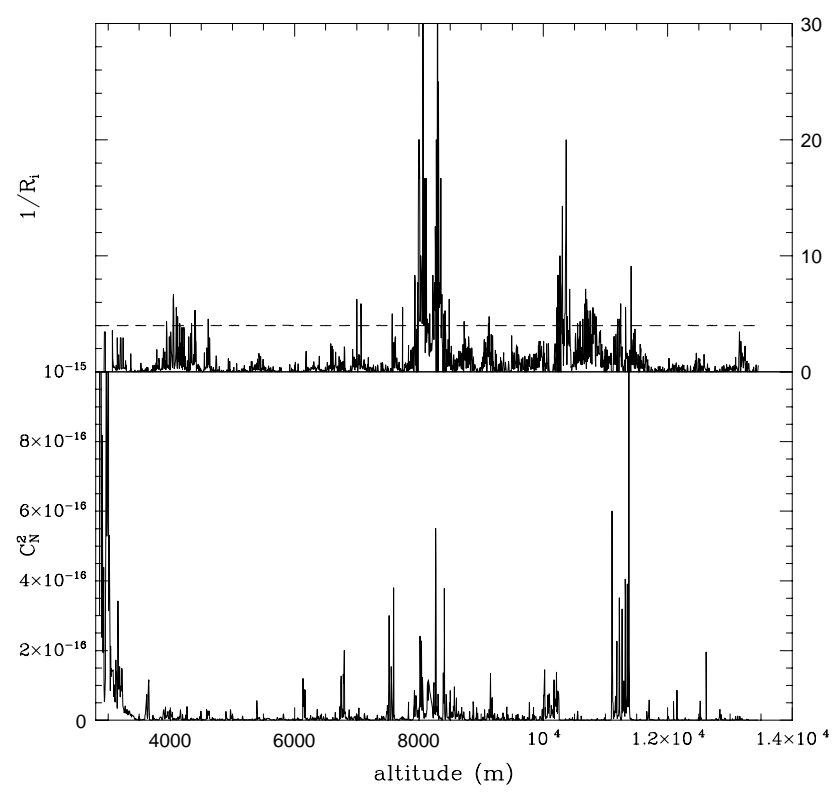

Fig. 6. A comparison of $C_{\mathrm{N}}^{2}$ and $R_{\mathrm{i}}$ profiles for $02 / 07 / 95.1 / R_{\mathrm{i}}$ is plotted to show more clearly the regions of instability: any peaks above the dashed line satisfy $R_{\mathrm{i}}<1 / 4$. The integrated seeing values calculated for this flight were: $\varepsilon_{\text {tot. }}=0.83^{\prime \prime}, \varepsilon_{\mathrm{FA}}=$ $0.41^{\prime \prime}$

show a significant boundary layer contribution to the overall seeing, it usually extends to at least $1-2 \mathrm{~km}$ above ground level, and is invariably of much lower intensity. In addition, upper atmosphere turbulence, arising from jet streams and temperature fluctuations in the tropopause, is often a major component of the seeing at these sites.

Table 3 summarises seeing measurements from some of the world's leading observatory sites. It can be seen quite clearly that the optical turbulence at the South Pole is concentrated much closer to the surface than at any of these sites. The free atmosphere contribution is comparable to or lower than the best quoted results from the

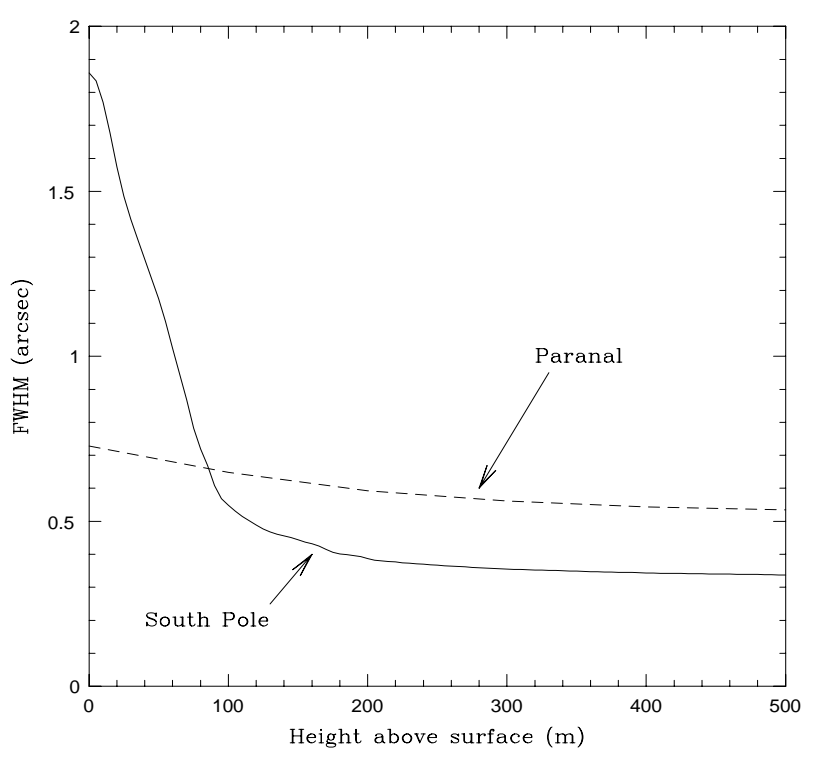

Fig. 7. Seeing as a function of height of the telescope above the surface. The solid line represents average results from our launches at the South Pole, while the dashed line is a summary of a similar experiment performed at the ESO-VLT site at Cerro Paranal, northern Chile (Fuchs 1995) in May 1993, averaged over 13 flights

other sites. It is important to note that the value of $0.37^{\prime \prime}$ is calculated upward from $\sim 200 \mathrm{~m}$ above the surface. The contribution from the atmosphere above $2000 \mathrm{~m}$ (the average boundary layer height at the mid-latitude sites of similar altitude) at the South Pole is $<0.3^{\prime \prime}$.

As a more direct comparison, Fig. 7 shows the derived seeing, averaged over the fifteen flights, as a function of the altitude at which a hypothetical telescope is placed. This plot is obtained by increasing the lower altitude limit of the integral in Eq. (3) in $5 \mathrm{~m}$ steps. The optical turbulence falls sharply up to an altitude of $\sim 120 \mathrm{~m}$, and decreases more gradually up to $200 \mathrm{~m}$, beyond which the 


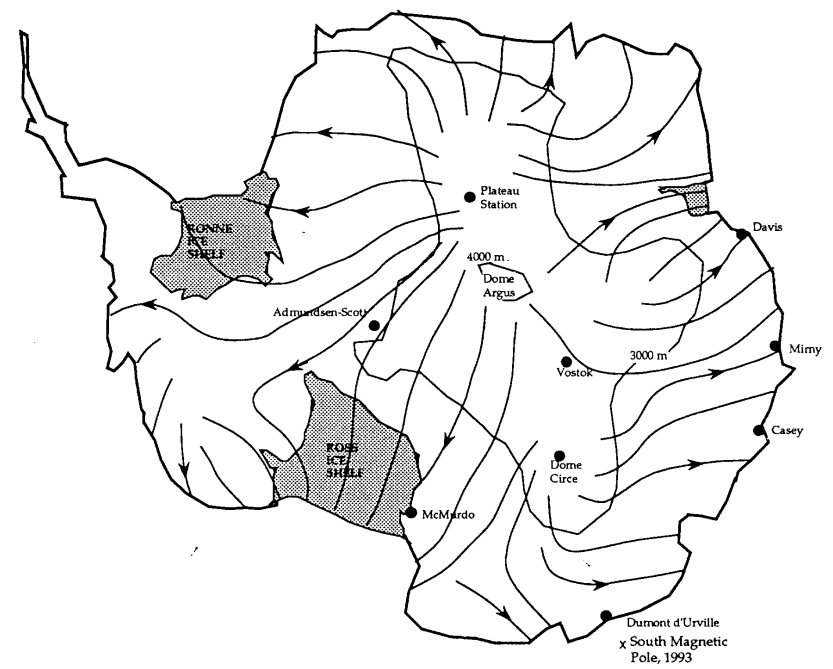

Fig. 8. Contour map of surface wind speeds over Antarctica, from Dopita 1993, based on results of Schwerdtfeger 1984

very low remaining seeing contribution decreases smoothly with altitude.

The results of similar experiments (averaged over thirteen flights) performed at the ESO-VLT site at Cerro Paranal, Northern Chile (Fuchs 1995), are included in the same figure. The two data sets were analysed using the same method. It is clear that, while South Pole is an inferior site (in terms of seeing) from ground level, most of the image degradation occurs very close to the surface. The seeing is better than that measured at Paranal above an altitude of only $\sim 100 \mathrm{~m}$. The free atmosphere contribution, i.e. above $\sim 250 \mathrm{~m}$, is around $60 \%$ of the corresponding value calculated from the Paranal data.

\section{Discussion}

\subsection{Other possible sites in Antarctica}

The South Pole does not lie on one of the highest points of the antarctic plateau, and is therefore not expected to represent the best seeing conditions available in Antarctica. The results obtained here lead us to consider the possibility that the $0.3-0.4^{\prime \prime}$ seeing measured above the $100-200 \mathrm{~m}$ high boundary layer might extend to ground level at other sites on the plateau.

The surface winds at the South Pole are of katabatic origin, due to the descent of cold air from the higher regions of the plateau. The strength and direction of these winds over the continent are largely dependent on the local topography, as illustrated in Fig. 6 (Dopita 1993; Schwerdtfeger 1984). The strong wind shears observed occur at the boundary between this katabatic flow and the upper atmosphere winds, which are generally geostrophic (Schwerdtfeger 1984). At Dome A $\left(4200 \mathrm{~m}, 82^{\circ} \mathrm{S} 80^{\circ} \mathrm{E}\right)$, Dome C $\left(3300 \mathrm{~m}, 74^{\circ} \mathrm{S} 123^{\circ} \mathrm{E}\right)$, and Dome F $(3810 \mathrm{~m}$, $77^{\circ} \mathrm{S} 40^{\circ} \mathrm{E}$ ), the three most significant local "peaks" on the antarctic plateau, these winds do not exist. Dome A, in particular, being the highest point on the plateau, is at the origin of the katabatic flow. It is likely that, at all of these sites, very little optical turbulence is produced in the boundary layer since, despite the possible presence of strong positive temperature gradients such as those measured at the South Pole, there is little mechanical mixing of the different temperature layers. If the free atmosphere turbulence at these sites is similar to that at the South Pole, we would expect to observe very good seeing from surface level: quite possibly the best seeing conditions available anywhere on the earth's surface.

In order to measure the important site characteristics at these locations, an automated astrophysical observatory (AASTO) has been built, which contains several instruments for measurement of the seeing and the atmospheric transmission over a wide range of wavelengths. The AASTO is designed to function independently for a full winter season, and is scheduled to be deployed at Dome C in 1999, and at Dome A in 2000.

The construction of a permanent station at Dome $\mathrm{C}$ has begun (the France/Italy Concordia Project), and is expected to be completed by 2000. This will enable the operation of a similar range of longer term site-testing experiments to those currently being conducted at the South Pole.

In addition, while there are currently no plans for astronomical site-testing at Dome F (also known as Dome Fuji), a temporary winter station is currently being operated there by Japanese scientists, whose experiments include meteorological balloon sondes, to be launched during 1997. Access to these data would provide some very enlightening comparisons to the measurements made at the South Pole.

\subsection{Other astrophysical parameters}

The measured $C_{\mathrm{N}}^{2}$ profiles allow calculation of several parameters necessary to determine the feasibility of image correction techniques (adaptive optics and speckle interferometry) at the site, as well as other quantities such as the scintillation index. Using the notation:

$\mathcal{C}(x, \eta)=\int x^{\eta}(h) C_{\mathrm{N}}^{2}(h) \mathrm{d} h, \quad \mathcal{C}(0)=\int C_{\mathrm{N}}^{2}(h) \mathrm{d} h$

these quantities are given by Eqs. (7-11) (Roddier 1981; Roddier et al. 1982). The subscripts AO and SI refer to adaptive optics and speckle interferometry respectively.

\section{Isoplanatic patch:}

$\theta_{\mathrm{AO}}=0.31 r_{0}\left(\frac{\mathcal{C}(h, 5 / 3)}{\mathcal{C}(0)}\right)^{-3 / 5}$
$\theta_{\mathrm{SI}}=0.36 r_{0}\left(\frac{\mathcal{C}(h, 2)}{\mathcal{C}(0)}-\left(\frac{\mathcal{C}(h, 1)}{\mathcal{C}(0)}\right)^{2}\right)^{-1 / 2}$ 
Table 3. Summary of the calculated values of the astrophysical parameters defined in Eqs. (7-11), based on the 15 balloon flights. The limit of the boundary layer is taken to be $220 \mathrm{~m}$. Values for Cerro Paranal were obtained using the average $C_{\mathrm{N}}^{2}$ profiles measured at this site (Fuchs 1995), although no wind data were available. Results for La Palma are from Vernin \& Tuñon-Muñóz (1994)

\begin{tabular}{lccccccccccc}
\hline Site & $\theta_{\mathrm{AO}}\left[^{\prime \prime}\right]$ & \multicolumn{2}{c}{$\theta_{\mathrm{SI}}\left[{ }^{\prime \prime}\right]$} & \multicolumn{2}{c}{$\tau_{\mathrm{AO}}[\mathrm{ms}]$} & $\tau_{\mathrm{SI}}[\mathrm{ms}]$ & \multicolumn{2}{c}{$\sigma_{\mathrm{I}}^{2}$} \\
& mean best & mean & best & mean & best & mean & best & mean best \\
\hline $\begin{array}{l}\text { South Pole } \\
\text {-total }\end{array}$ & 3.23 & 6.50 & 2.76 & 5.17 & 1.58 & 6.09 & 17.34 & 35.04 & 0.070 & 0.033 \\
- B.L. correction & 65.35 & - & 118.22 & - & 1.62 & - & 17.81 & - & - & - \\
Cerro Paranal & 1.45 & - & 1.88 & - & - & - & - & - & 0.16 & - \\
La Palma & 1.30 & 1.62 & 2.18 & 2.66 & 6.64 & 9.60 & 12.88 & 19.52 & - & - \\
\hline
\end{tabular}

\section{Coherence time:}

$$
\begin{aligned}
& \tau_{\mathrm{AO}}=0.31 r_{0}\left(\frac{\mathcal{C}(U, 5 / 3)}{\mathcal{C}(0)}\right)^{-3 / 5} \\
& \tau_{\mathrm{SI}}=0.36 r_{0}\left(\frac{\mathcal{C}(U, 2)}{\mathcal{C}(0)}-\left(\frac{\mathcal{C}(U, 1)}{\mathcal{C}(0)}\right)^{2}\right)^{-1 / 2}
\end{aligned}
$$

Scintillation index:

$$
\sigma_{\mathrm{I}}^{2}=19.12 \lambda^{-7 / 6} \mathcal{C}(h, 5 / 6) \text {. }
$$

\subsubsection{Isoplanatic patch, coherence time}

The isoplanatic angle, $\theta$, depends on the altitude distribution of the turbulent cells producing the seeing, according to Eqs. (7-8). This angle represents the maximum angular distance between the source of interest and a reference star for which the wavefront distortions are coherent and may, in principle, be fully corrected. The maximum integration time, $\tau$, for which a given correction remains accurate is limited by temporal isoplanatism, due to the movement of turbulent cells across the field of view at the wind velocity, according to Eqs. (9-10). The values of these quantities, calculated from the measured $C_{\mathrm{N}}^{2}$ profiles are shown in Table 3, along with corresponding values from La Palma and Cerro Paranal. The results at the South Pole are somewhat better than at the other sites, especially during the best observing conditions. The improvement is minor, however, especially in the values of $\theta_{\mathrm{AO} / \mathrm{SI}}$, and does not greatly relieve the problem that the probability of finding a suitable reference source within this area of the sky is extremely low $\left(\sim 10^{-3}\right.$ at visual magnitude $\left.m_{v}=16\right)$. The relatively large difference between $\tau_{\mathrm{SI}}$ and $\tau_{\mathrm{AO}}$ is due to the different $C_{\mathrm{N}}^{2}$ and $\boldsymbol{U}$ profiles, compared with other sites, as discussed in Sect. 3.4.

In practice, varying degrees of partial correction may be sought, and the performance of such systems has been analysed in detail (e.g. Cowie \& Songaila 1988; Wilson et al. 1996). Evidently, the maximum image "reconstruction angle" (the term coined by Cowie \& Songaila) depends on the degree of correction required. A trade-off must be made between the quality of the corrected image and access to enough suitable reference sources to obtain adequate sky coverage.

As discussed previously, the large majority of the optical turbulence at the South Pole occurs in the lowest $\sim 200 \mathrm{~m}$. Thus, and due to the strong height dependence of $\theta_{\mathrm{AO} / \mathrm{SI}}$ shown in Eqs. (7-8), we may expect that an image correction system that removed the boundary layer component of the seeing, leaving the remainder uncorrected, would be effective over a substantially greater area of the sky than such a system operating at the best mid-latitude sites.

In a simple approximation, angles for partial image correction were calculated by varying the upper limits, $h_{\mathrm{u}}$, of the integrals in Eqs. (7-8). The residual seeing, $\varepsilon_{\mathrm{r}}$, related to each of these angles, may be found by setting $h_{\mathrm{u}}=h_{0}$ in Eq. (3), and using the values calculated in Sect. 3.4 (Fig. 7). As expected, the reconstruction angle increases rapidly for corrections limited to the boundary layer seeing (i.e. $h \simeq 200 \mathrm{~m}, \varepsilon_{\mathrm{r}} \simeq 0.3^{\prime \prime}$ on average), as shown in Table $3\left(\theta_{\mathrm{SI}}=1^{\prime} 58^{\prime \prime}, \theta_{\mathrm{AO}}=1^{\prime} 6^{\prime \prime}\right)$, and Fig. 9. A system operating at $\varepsilon_{\mathrm{r}}=0.3^{\prime \prime}$ at Cerro Paranal would require that $h_{\mathrm{u}}$ be much higher (see Fig. 7), resulting in substantially smaller reconstruction angles $\left(\theta_{\mathrm{SI}}=13^{\prime \prime}\right.$, $\left.\theta_{\mathrm{AO}}=10^{\prime \prime}\right)$. Thus, the difference between the sites at the same level of partial image correction is very large.

The improvement in terms of sky coverage associated with the increased partial correction angles can be found by determining the probability of finding an appropriate guide star of magnitude $m_{v}$ within an angle $\theta$, at Galactic latitude $b$ (Olivier et al. 1993):

$P_{\text {sky }}=1-\exp \left[-\pi \theta^{2} \Sigma\left(m_{v}, b\right)\right]$

where $\Sigma\left(m_{v}, b\right)$ is the number density of stars of a given $m_{v}$ and $b$. Values of $\Sigma\left(m_{v}, b\right)$ are tabulated in various astronomical data books (e.g. Allen 1973).

From Fig. 9 and Eq. (12), we have a relationship between $P_{\text {sky }}$ and the uncorrected component of the seeing. Figures 10a,b shows this relationship for various values of $m_{v}$ averaged across all galactic latitudes, for both speckle interferometry and adaptive optics. Corresponding 

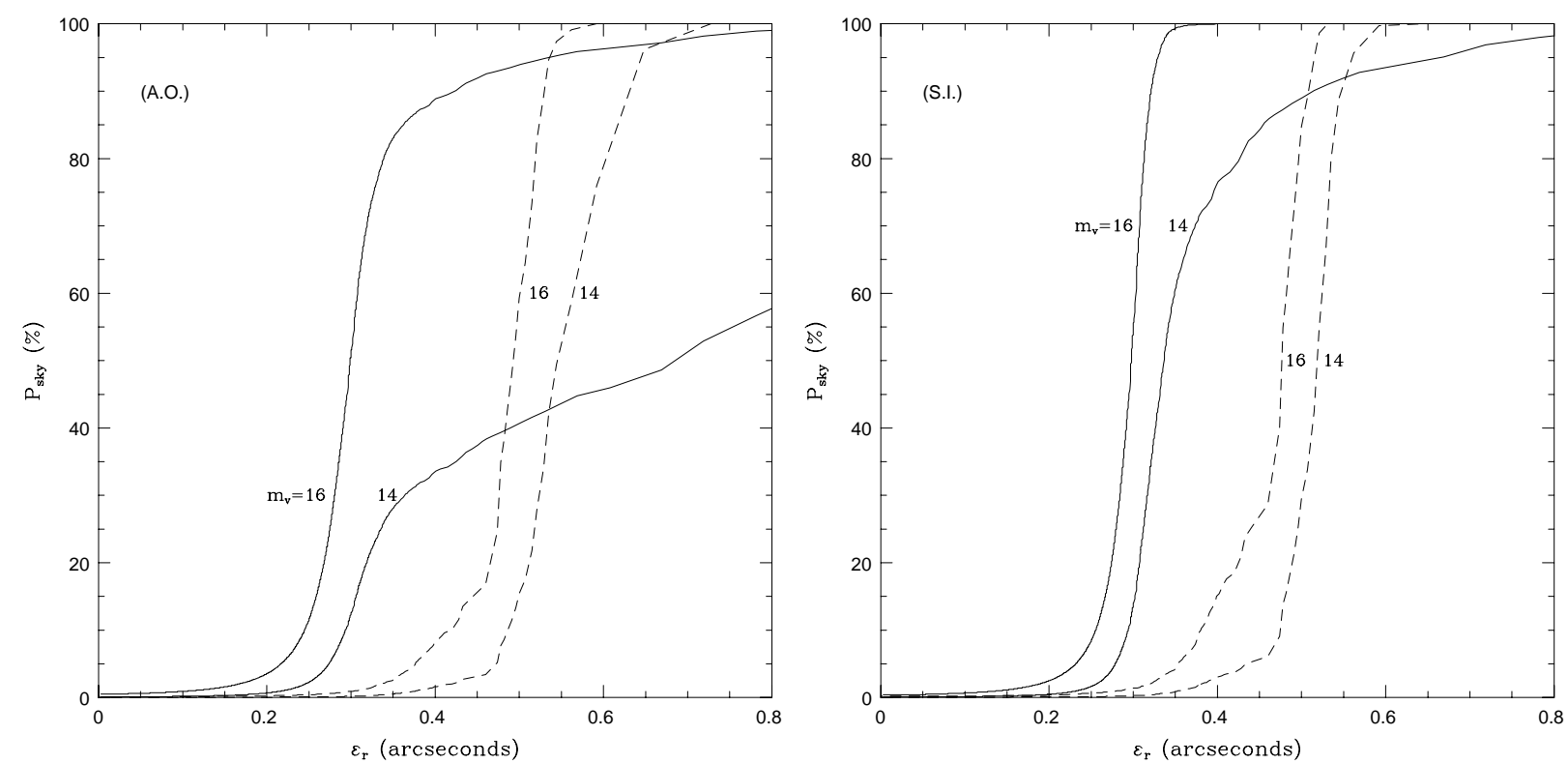

Fig. 10a and b. Percentage sky coverage as a function of the residual seeing, for a) adaptive optics and b) speckle interferometry, in the visual magnitude range $m_{v}=14-16$. Solid lines show the results for the South Pole; dashed lines for Cerro Paranal. Calculations are averaged over all galactic latitudes

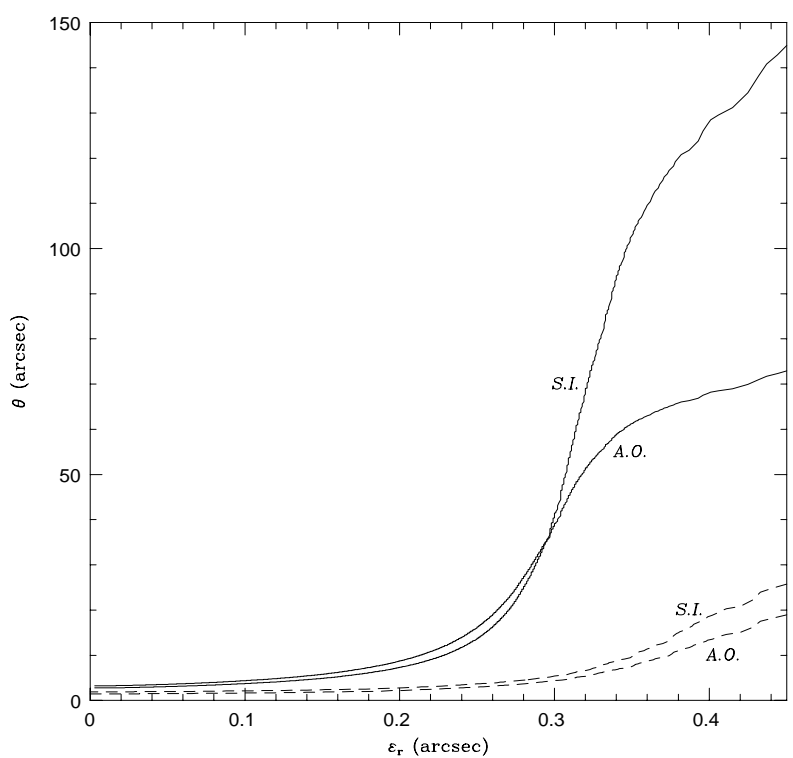

Fig. 9. $\theta_{\mathrm{AO} / \mathrm{SI}}$, as a function of the residual seeing $\varepsilon_{\mathrm{r}}$. Solid lines are derived from the average $C_{\mathrm{N}}^{2}$ profiles at the South Pole, and the dashed lines from the Cerro Paranal data. The curves are obtained by varying the upper and lower limits of Eqs. (7)(8) and Eq. (3), respectively. The value of $\theta$ for $\varepsilon=0$ is the isoplanatic angle

calculations based on the available data from Cerro Paranal are included for comparison.

Figure 10 shows that the sky coverage is up to around $75 \%$ for image quality $\varepsilon_{\mathrm{r}}=0.3^{\prime \prime}$, using reference sources in the range $m_{v}=16$ to $m_{v}=14$. This value decreases somewhat at the bright end, in the adaptive optics case
(Fig. 10a). The percentage of sky covered at Cerro Paranal over this range of $\varepsilon_{\mathrm{r}}$ and $m_{v}$ is around $1-2 \% .75 \%$ sky coverage would be possible at this site only with images in the range $\varepsilon_{\mathrm{r}}=0.4-0.5^{\prime \prime}$.

Note that these values are calculated for an "average" number density of stars at each magnitude; the higher density near the galactic equator improves the resolution by $\sim 0.05^{\prime \prime}$ for a given value of $P_{\text {sky }}$. A further improvement of approximately the same magnitude should also be obtained in the infrared, bringing the residual seeing down to the range $\varepsilon_{\mathrm{r}} \sim 0.1-0.2^{\prime \prime}$.

As noted above, these results assume that partial corrections may be performed based directly on the altitude distribution of the turbulent layers. This is not possible in practice, but, rather, any method of partial correction must take into account the power spectrum of the turbulence. However, the comparison between the two sites certainly remains valid, and the results are a good qualitative indication of the attainable image resolution at the site.

Some of the practical methods that have been developed to increase the workable angle of adaptive optics systems indicate that the achievable image quality may be significantly better than the values stated here. Cowie \& Songaila (1988) were able to obtain $0.1-0.2^{\prime \prime}$ images at Mauna Kea over an angle of up to $\sim 30^{\prime \prime}$ (a factor of five greater than the isoplanatic angle at this site) by relaxing the requirement of full isoplanicity at high frequencies.

Another method that has been investigated is the "multiconjugate" approach (e.g. Tallon et al. 1992), in which a number of deformable mirrors are used, each placed conjugate with a turbulent layer. Theoretical 
calculations have been performed by Wilson \& Jenkins (1996), to determine the relative performance of image correction systems using pupil and turbulence conjugation. It was found that the sky coverage was greater using turbulence conjugation, compared with pupil conjugation, by a factor of $2-3$ (at the same level of image correction).

Turbulence conjugation is a particularly powerful technique in situations where the bulk of the image degradation is concentrated in a small number of turbulent layers, which is clearly seen to be the case at the South Pole (Figs. 4a-f). Since most of the boundary layer turbulence is confined to $\sim 1-3$ intense turbulent layers, with perhaps $2-3$ weaker layers throughout the free atmosphere, it can be envisaged that such a system operating at this site would be capable of very high resolution imagery over a large proportion of the sky.

\subsubsection{Scintillation index}

The scintillation index, $\sigma_{\mathrm{I}}^{2}$ (Eq. (11)), is a measure of the amplitude fluctuations in the received signal from an astronomical source, caused by atmospheric turbulence. It is an important limiting factor in observations that measure small variations in the flux of a source, including observations of variable stars, and asteroseismology. The $h^{5 / 6}$ dependence of $\sigma_{\mathrm{I}}^{2}$ indicates that this quantity, too, should have a lower value at the South Pole than at any other site.

The results for $\lambda=0.5 \mu \mathrm{m}$, summarised in Table 3 and Fig. 11, show that the average value of $\sigma_{\mathrm{I}}^{2}$ is about $40 \%$ of the corresponding value calculated from the Cerro Paranal data (which, again, represents close to the best conditions measured at a mid-latitude site). These results, together with the long continuous observations possible during the polar night, indicate that the antarctic plateau is an ideal site for the types of observations mentioned above.

\section{Conclusion}

The atmospheric seeing at the South Pole has been measured during a campaign of 15 balloon launches from June-August 1995. The vertical $C_{\mathrm{N}}^{2}$ profiles indicate the presence of an highly turbulent boundary layer up to an altitude of $100-250 \mathrm{~m}$, with a very stable free atmosphere.

The boundary layer turbulence is closely associated with the katabatic wind and surface temperature inversion (Fig. 5). This suggests that the seeing at other sites higher on the plateau, where the katabatic wind is absent, is likely to be substantially lower than the average $1.86^{\prime \prime}$ $\left(1.36^{\prime \prime}\right.$ at $\left.2.4 \mu \mathrm{m}\right)$ from surface level at the Pole, and may well approach the $0.37^{\prime \prime}\left(0.27^{\prime \prime}\right.$ at $\left.2.4 \mu \mathrm{m}\right)$ free atmosphere contribution. This would be significantly better than any other site studied to date, and, combined with the excellent atmospheric transmission at infrared wavelengths,

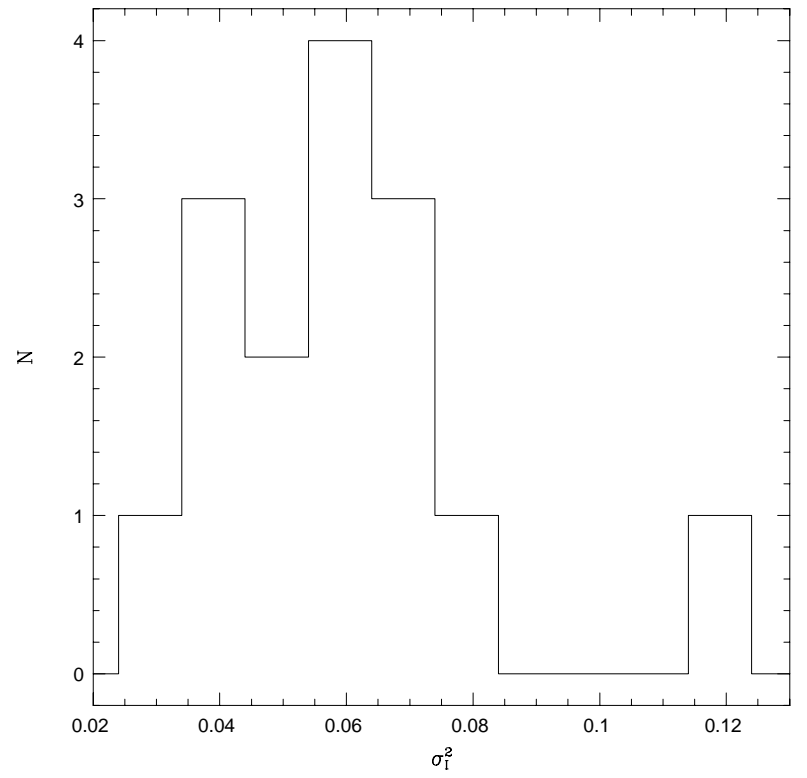

Fig. 11. Distribution of the value of $\sigma_{\mathrm{I}}^{2}$ calculated for each of the fifteen balloon flights. The highest measured value, $\sigma_{\mathrm{I}}^{2}=$ 0.118 , is statistically more than $3 \sigma$ above the mean, and the $C_{\mathrm{N}}^{2}$ data from this flight were omitted when calculating the average value shown in Table 3

the low scintillation index, and the possibility of performing long continuous observations, the potential scientific value of an appropriate observatory on the high antarctic plateau is undeniable. It is, of course, necessary to quantify the atmospheric seeing and transmission characteristics of sites such as Domes A and C, and such measurements are planned for the near future.

The vertical $C_{\mathrm{N}}^{2}$ profile at the South Pole is favourable for image restoration using an adaptive optics system that corrects for the effects of the boundary layer turbulence. At optical wavelengths, image resolution of $\lesssim 0.2^{\prime \prime}$ FWHM should be attainable over an angle of $2^{\prime}$ or more, which would provide excellent sky coverage even for reasonably bright reference sources. If this level of performance is found to be practically achievable, it may be that the South Pole itself would be a suitable observatory site, given the support infrastructure already in place at this location.

Acknowledgements. We wish to thank Al Harper, Bob Pernic and Bob Loewenstein from CARA (the University of Chicago), and John Storey, Michael Ashley and Michael Burton from the University of New South Wales, for their assistance. This research was supported in part by the U.S.A. National Science Foundation under a cooperative agreement with the Center for Astrophysical Research in Antarctica (CARA), grant number NSF OPP 89-20223. CARA is a National Science Foundation Science and Technology Center. Support from the Australian Department of Industry Science and Technology's Bilateral Program is gratefully acknowledged. Funding for all instrumentation was provided by l'Institut National des Sciences de l'Univers, France. We make special mention of the late 
Jean-François Manigault for his part in the successful completion of this research.

\section{References}

ESO-VLT working group on site evaluation, 1987, VLT report No. 55, M. Sarazin (ed.)

Allen C.W., 1973, Astrophysical Quantities. Athlone Press

Ashley M.C.B., Burton M.G., Lloyd J.P., Storey J.W.V., 1995, SPIE 2552, 33

Ashley M.C.B., Burton M.G., Storey Lloyd J.P., et al., 1996, PASP 108, 721

Bally J., Theil D., Billawala Y., 1996, Proc. Astron. Soc. Aust. 13, 22

Burton M.G., Aitken D.K., Allen D.A., et al., 1994, Proc. Astron. Soc. Aust. 11, 127

Burton M.G., 1996, Pub. Astron. Soc. Aust. 13, 2

Cowie L.L., Songaila A., 1988, J. Opt. Soc. Am. 5, 1015

Dierickx P., 1992, J. Mod. Opt. 39, 569

Dopita M.A., 1993, IABO: The International Antarctic Balloon Observatory (draft copy)

Forbes F.F., 1989, SPIE 1114, 28

Fried D.L., 1966, J. Opt. Soc. Am. 56, 1372

Fuchs A., 1995, Contribution à l'étude de l'apparition de la turbulence optique dans les couches minces. Concept du SCIDAR generalise (Ph. D. Thesis), Université de NiceSophia Antipolis, France

Gillingham P.R., 1993, ANARE Res. Notes 88, 290, Australian Institute of Physics 10th Congress, University of Melbourne, February 1992 (publications of the Antarctic Division)

Marks R.D., Vernin J., Azouit M., et al., 1996, A\&AS 118, 385

Murtagh F., Sarazin M., 1993, PASP 105, 932
Neff W.D., 1981, An Observational and Numerical Study of the Atmospheric Boundary Layer Overlying the East Antarctic Ice Sheet (Ph. D. thesis), Wave Propagation Laboratory, Boulder Colorado, U.S.A.

Nguyen H.T., Rauscher B.J., Severson S.A., et al., 1996, PASP 108,718

Obukhov A.M., 1949, Izv. Akad. Nauk SSSR, Ser Geograf. Geofis. 13, 58

Olivier S.S., Max C.E., Gavel D.T., Brase J.M., 1993, ApJ 407, 428

Parenti R.R., 1992, Lin. Lab. J. 5, 93

Roddier F., 1981, Prog. Opt. 19, 281

Roddier F., Gilli J.M., Lund G., 1982, J. Opt. Paris 13, 263

Roddier F., Cowie L., Graves J.E., et al., 1990, SPIE 1236, 485

Sarazin M., Roddier F., 1990, A\&A 227, 294

Schwerdtfeger W. (ed.), 1984, Weather and Climate of the Antarctic. Elsevier Science Pub. Co. NY

Storey J.W.V., Ashley M.C.B., Burton M.G., 1995, Publ. Astron. Soc. Aust. 13, 35

Tallon M., Foy R., Vernin J., 1992, Laser guide star adaptive optics workshop, Fugate R.Q. (ed.), Albuquerque (10-12 March 1992)

Tatarski V.I., 1961, Wave Propagation in a Turbulent Medium. McGraw-Hill, New York

Vernin J., 1994, Recherche de site pour l'astronomie en Antarctique, Colloque Acad. Sci. Paris, 16-18 Dec. 1992, 92-96

Vernin J., Marks R., Ashley M.C.B., et al., 1994, Optical Turbulence at the South Pole: First Measurements and Future Plans, XXIIIrd SCAR Meeting, Rome, Aug. 29-Sep. 1

Vernin J., Muñon-Tuñóz C., 1992, A\&A 257, 811

Vernin J., Muñon-Tuñóz C., 1994, A\&A 284, 311

Wilson R.W., Jenkins C.R., 1996, MNRAS 268, 39 